\title{
Cosmic-ray acceleration in young protostars
}

\author{
M. Padovani ${ }^{1,2}$, P. Hennebelle ${ }^{3}$, A. Marcowith ${ }^{1}$, and K. Ferrière ${ }^{4}$ \\ ${ }^{1}$ Laboratoire Univers et Particules de Montpellier, UMR 5299 du CNRS, Université de Montpellier, place E. Bataillon, cc072, \\ 34095 Montpellier, France \\ e-mail: Marco.Padovani@umontpellier .fr \\ 2 INAF-Osservatorio Astrofisico di Arcetri, Largo E. Fermi 5, 50125 Firenze, Italy \\ 3 CEA, IRFU, SAp, Centre de Saclay, 91191 Gif-Sur-Yvette, France \\ 4 IRAP, Université de Toulouse, CNRS, 9 avenue du Colonel Roche, BP 44346, 31028 Toulouse Cedex 4, France
}

Received 1 July 2015 / Accepted 21 September 2015

\section{ABSTRACT}

\begin{abstract}
The main signature of the interaction between cosmic rays and molecular clouds is the high ionisation degree. This decreases towards the densest parts of a cloud, where star formation is expected, because of energy losses and magnetic effects. However recent observations hint to high levels of ionisation in protostellar systems, therefore leading to an apparent contradiction that could be explained by the presence of energetic particles accelerated within young protostars. Our modelling consists of a set of conditions that has to be satisfied in order to provide an efficient particle acceleration through the diffusive shock acceleration mechanism. We find that jet shocks can be strong accelerators of protons which can be boosted up to relativistic energies. Another possibly efficient acceleration site is located at protostellar surfaces, where shocks caused by impacting material during the collapse phase are strong enough to accelerate protons. Our results demonstrate the possibility of accelerating particles during the early phase of a proto-Solar-like system and can be used as an argument to support available observations. The existence of an internal source of energetic particles can have a strong and unforeseen impact on the star and planet formation process as well as on the formation of pre-biotic molecules.
\end{abstract}

Key words. cosmic rays - ISM: jets and outflows - stars: protostars

\section{Introduction}

It is largely accepted that Galactic cosmic rays, which pervade the interstellar medium, are likely produced in shock waves in supernova remnants (Drury 1983). Cosmic rays activate the rich chemistry that is observed in a molecular cloud (Duley \& Williams 1984), and also regulate its collapse timescale (Balbus \& Hawley 1991; Padovani et al. 2014), determining the efficiency of star and planet formation. However, cosmic rays cannot penetrate the densest parts of a molecular cloud, where the formation of stars is expected, as a result of energy losses and magnetic field deflections (Padovani et al. 2009, 2013; Padovani \& Galli 2011, 2013; Cleeves et al. 2013). Recently, observations towards young protostellar systems show a surprisingly high value of the ionisation rate (Ceccarelli et al. 2014; Podio et al. 2014), the main indicator of the presence of cosmic rays in molecular clouds. Synchrotron emission, the typical feature of relativistic electrons, was also detected towards the bow shock of a T Tauri star (Ainsworth et al. 2014). Nevertheless, the origin of these signatures unique to energetic particles is still puzzling. Here we show that particle acceleration can be driven by shock waves occurring within protostars.

\section{Particle acceleration in shocks}

This works focuses on shock acceleration by means of the diffusive shock acceleration (DSA) mechanism. Also known as firstorder Fermi acceleration, DSA is a process where charged particles systematically gain energy while crossing a shock front. Multiple shock crossings allow the particle energy to rapidly increase, reaching the relativistic domain. The motion of particles back and forth from upstream to downstream requires the presence of magnetic fluctuations that produce a scattering of the pitch angle, namely the angle between the particle's velocity and the mean magnetic field (Drury 1983; Kirk 1994). We argue below that the accelerated particles themselves can produce the necessary fluctuations to maintain DSA around shocks, as discussed e.g. in Bell (1978).

In the following subsections, we describe all the conditions that have to be satisfied to effectively accelerate protons and electrons through DSA. The acceleration of helium and heavier nuclei will be presented in a subsequent paper. All the constraints limiting the maximum energy of the accelerated particles are written as functions of the upstream flow velocity in the shock reference frame, $U_{\mathrm{sh}}=v_{\mathrm{fl}}-v_{\mathrm{sh}}, v_{\mathrm{fl}}$ and $v_{\mathrm{sh}}$ are the flow and the shock velocities in the observer reference frame, respectively. Our aim is to compute the maximum energy, $E_{\max }$, reached by a particle in the regime where ions and neutrals are coupled. As explained in Sect. 2.3, in this case the damping of the Alfvén waves, which determines the confinement of particles, is weak and particle acceleration is more effective.

\subsection{Condition on shock velocity}

In order to have efficient particle acceleration, the flow has to be supersonic and super-Alfvénic. These two conditions are combined into the following relation

$U_{\mathrm{sh}, 2}>\max \left\{9 \times 10^{-2}\left[\gamma_{\mathrm{ad}} T_{4}(1+x)\right]^{0.5}, 2 \times 10^{-4} n_{6}^{-0.5} B_{-5}\right\}$, 
where $U_{\mathrm{sh}, 2}$ has units of $10^{2} \mathrm{~km} \mathrm{~s}^{-1}, \gamma_{\mathrm{ad}}$ is the adiabatic index, $T_{4}$ the upstream temperature in $10^{4} \mathrm{~K}, n_{6}$ the total number density of hydrogen in $10^{6} \mathrm{~cm}^{-3}, x$ the ionisation fraction, and $B_{-5}$ the magnetic field strength in $10^{-5} \mathrm{G}$. The two terms in square brackets on the right-hand side of Eq. (1) are the ambient (or upstream) sound speed and the Alfvén speed of the total gas in $10^{2} \mathrm{~km} \mathrm{~s}^{-1}$, respectively.

\subsection{Condition on low-energy particle acceleration: collisional losses}

We are interested in the acceleration of low-energy particles ( $\lesssim 100 \mathrm{MeV}-1 \mathrm{GeV}$ ) because they are responsible for the bulk of the ionisation. We have to verify that the shock acceleration rate is larger than the collisional loss rate $\left(t_{\mathrm{acc}}^{-1}>t_{\text {loss }}^{-1}\right)$. Following Drury et al. (1996), the acceleration rate is given by

$t_{\mathrm{acc}}^{-1}=\frac{3.2 \times 10^{-8}}{\gamma-1} \frac{k_{\mathrm{u}}^{\alpha}(r-1)}{r\left(1+r k_{\mathrm{d}} / k_{\mathrm{u}}\right)} \tilde{\mu}^{-1} U_{\mathrm{sh}, 2}^{2} B_{-5} \mathrm{~s}^{-1}$,

where $\tilde{\mu}=m / m_{\mathrm{p}}$ is the particle mass normalised to the proton mass, $k_{\mathrm{u}}$ and $k_{\mathrm{d}}$ are the diffusion coefficients in the upstream and downstream media, respectively, normalised to the Bohm value for protons,

$k_{\mathrm{u}, \mathrm{d}}^{-\alpha}=\frac{\kappa_{\mathrm{u}, \mathrm{d}}}{\kappa_{\mathrm{B}}}=\frac{3 e B}{\gamma \beta^{2} m_{\mathrm{p}} c^{3}} \kappa_{\mathrm{u}, \mathrm{d}}$,

where $\gamma$ is the Lorentz factor, $\beta=\gamma^{-1} \sqrt{\gamma^{2}-1}$, and $r$ is the shock compression ratio. For a parallel shock, $\alpha=-1$ and $\kappa_{\mathrm{u}}=\kappa_{\mathrm{d}}$, while for a perpendicular shock, $\alpha=1$ and $\kappa_{\mathrm{u}}=r \kappa_{\mathrm{d}}{ }^{1}$. The energy loss rate is given by

$t_{\mathrm{loss}}^{-1}=3.2 \times 10^{-9} \frac{\beta}{\gamma-1} \tilde{\mu}^{-1} n_{6} L_{-25} \mathrm{~s}^{-1}$,

where $L_{-25}$ is the energy loss function (Padovani et al. 2009) in units of $10^{-25} \mathrm{GeV} \mathrm{cm}{ }^{2}$ which was extended to lower energies including Coulomb losses (for protons, Mannheim \& Schlickeiser 1994; and electrons, Swartz et al. 1971) and synchrotron losses (Schlickeiser 2002). The maximum energy of accelerated particles set by energy losses, $E_{\text {loss }}$, is found when $t_{\mathrm{acc}}^{-1}=t_{\mathrm{loss}}^{-1}$, specifically

$\beta L_{-25}=10 \frac{k_{\mathrm{u}}^{\alpha}(r-1)}{r\left(1+r k_{\mathrm{d}} / k_{\mathrm{u}}\right)} U_{\mathrm{sh}, 2}^{2} n_{6}^{-1} B_{-5}$.

\subsection{Condition on particle acceleration: ion-neutral friction}

The main limit on the possibility of particle acceleration is given by the presence of an incomplete ionised medium. In fact, the collision rate between ions and neutrals can be as high as to decrease the effectiveness of the DSA, damping the particle's self-generated Alfvén waves responsible for the particle scattering the shock back and forth. Ions and neutrals are effectively decoupled if the wave frequency is larger than the ion-neutral collision frequency. Following Drury et al. (1996) and accounting for the fact that particles are not fully relativistic, we find that the critical energy separating these two regimes, $E_{\text {coup }}$, is derived by solving the following relation

$\gamma \beta=8.5 \times 10^{-7} \tilde{\mu}^{-1} T_{4}^{-0.4}\left(n_{6} x\right)^{-1.5} B_{-5}^{2}$.

\footnotetext{
1 A parallel/perpendicular shock is when the shock normal is parallel/perpendicular to the ambient magnetic field, respectively.
}

If the particle energy is larger than $E_{\text {coup }}$, ions and neutrals are coupled.

The upper cut-off energy due to wave damping, $E_{\mathrm{damp}}$, is set by requiring that the flux of accelerated particles advected downstream by the flow is equal to the flux of particles lost upstream because of the lack of waves (due to wave damping) to confine the particles. Following Drury et al. (1996), using their exact equation for the wave damping rate, accounting for departures from fully relativistic behaviour, and assuming $U_{\text {sh }}$ to be much larger than the Alfvén speed, $E_{\text {damp }}$ follows from

$\gamma \beta^{2}=8.8 \times 10^{-5} \tilde{\mu}^{-1} \Xi U_{\mathrm{sh}, 2}^{3} T_{4}^{-0.4} n_{6}^{-0.5}(1-x)^{-1} B_{-5}^{-4} P_{-2}^{\prime}$,

where

$\Xi=B_{-5}^{4}+1.4 \times 10^{12} \tilde{\mu}^{2} \gamma^{2} \beta^{2} T_{4}^{0.8} n_{6}^{3} x^{2}$.

Both Eqs. (6) and (7) are valid for $T \in\left[10^{2}, 10^{5}\right] \mathrm{K}$. The parameter $P_{-2}^{\prime}$ is the fraction of the shock energy $\left(m_{p} n U_{\mathrm{sh}}^{2}\right)$ going into particle acceleration in $10^{-2}$, which is proportional to the shock efficiency $\eta \in\left[10^{-6}, 10^{-3}\right]$ (Bykov 2004) and represents the fraction of particles extracted from the thermal plasma that is injected into the acceleration process by a shock. We predict both non-relativistic and mildly relativistic accelerated particles and we checked a posteriori that there is no strong back reaction. This means that the upstream medium is not warned by these particles that a shock is coming and we can safely assume that the shock and DSA process are unmodified. In other words, calculations are carried out in the test-particle limit.

If $E_{\text {damp }}>E_{\text {coup }}$, then $E_{\text {damp }}$ is in the coupled regime, namely neutrals coherently move with ions and ion-generated waves are weakly damped. The last inequality can be written by combining Eqs. (6) and (7) as

$\mathscr{R}=\frac{10^{2}}{\beta} \Xi U_{\mathrm{sh}, 2}^{3} n_{6} x^{1.5}(1-x)^{-1} B_{-5}^{-6} P_{-2}^{\prime}>1$.

We consider shocks in three types of environments: in jets as well as in accretion flows in the collapsing envelopes and on the surfaces of protostars. Using the range of parameters of Table 1, we find that $\mathscr{R} \ll 1$ in protostellar envelopes (Sect. 3 ). This is to say that the following two conditions on shock age and geometry (Sect. 2.4) are only discussed with reference to shocks in jets and on protostellar surfaces.

\subsection{Conditions due to shock age and geometry}

The maximum energy set by the age of the shock, $E_{\text {age }}$, is found when the acceleration time, given by the inverse of Eq. (2), is equal to the age of the shock. The latter can be assumed of the order of the dynamical time of the jet $\left(\gtrsim 10^{3} \mathrm{yr}\right.$, de Gouveia Dal Pino 1995) or equal to the accretion time in the case of a surface shock ( $\sim 10^{5} \mathrm{yr}$, Masunaga $\&$ Inutsuka 2000$)$. Then, $E_{\text {age }}$ is computed from

$\gamma-1=10^{3} \frac{k_{\mathrm{u}}^{\alpha}(r-1)}{r\left(1+r k_{\mathrm{d}} / k_{\mathrm{u}}\right)} \tilde{\mu}^{-1} U_{\mathrm{sh}, 2}^{2} B_{-5} t_{\mathrm{age}, 3}$,

with $t_{\text {age, } 3}$ in units of $10^{3} \mathrm{yr}$.

A further constraint is given by the geometry of the shock. In particular, the upstream diffusion length, $\lambda_{\mathrm{u}}=\kappa_{\mathrm{u}} / U_{\mathrm{sh}}$, has to be at most a given fraction $\epsilon<1$ of the shock radius, $R_{\mathrm{sh}}$; besides, in the jet configuration particles may also escape in the transverse direction. The shock can be assumed to be planar as long as the particle's mean free path around the shock is smaller 
Table 1. Values of the parameters described in the text.

\begin{tabular}{cccccc}
\hline \hline Site $^{*}$ & $\begin{array}{c}U_{\mathrm{sh}} \\
{\left[\mathrm{km} \mathrm{s}^{-1}\right]}\end{array}$ & $\begin{array}{c}T \\
{[\mathrm{~K}]}\end{array}$ & $\begin{array}{c}n \\
{\left[\mathrm{~cm}^{-3}\right]}\end{array}$ & $x$ & $\begin{array}{c}B \\
{[\mathrm{G}]}\end{array}$ \\
\hline $\mathcal{E}$ & $1-10$ & $50-100$ & $10^{7}-10^{8}$ & $\lesssim 10^{-6}$ & $10^{-3}-10^{-1}$ \\
$\mathcal{J}$ & $40-160$ & $10^{4}-10^{5}$ & $10^{4}-10^{7}$ & $0.01-0.9$ & $5 \times 10^{-5}-10^{-3}$ \\
$\mathcal{S}$ & 260 & $9.4 \times 10^{5}$ & $1.9 \times 10^{12}$ & $0.01-0.9$ & $10^{-1}-10^{3}$ \\
\hline
\end{tabular}

Notes. ${ }^{(*)} \mathcal{E}=$ envelope; $\mathcal{T}=$ jet $\mathcal{S}=$ protostellar surface.

than the transverse size of the jet, $R_{\perp}$. The maximum energy due to upstream escape losses, $E_{\mathrm{esc}, \mathrm{u}}$, follows from

$\gamma \beta^{2}=4.8 k_{\mathrm{u}}^{\alpha} \tilde{\mu}^{-1} U_{\mathrm{sh}, 2} B_{-5} \min \left(\epsilon R_{\mathrm{sh}, 2}, R_{\perp, 2}\right)$,

where both $R_{\mathrm{sh}, 2}$ and $R_{\perp, 2}$ are in units of $10^{2}$ AU. In the following we assume $\epsilon=0.1$ (Berezhko et al. 1996). Since jet shocks have a small transverse dimension, there is a further condition for the escape of particles downstream: the maximum energy due to downstream escape losses, $E_{\text {esc,d }}$, is found when the acceleration time, inverse of Eq. (2), is equal to the downstream diffusion time, $t_{\mathrm{diff}, \mathrm{d}}=R_{\perp}^{2} /\left(4 \kappa_{\mathrm{d}}\right)^{2}$, namely

$\gamma \beta^{2}(\gamma-1)=5.8 \frac{\left(k_{\mathrm{u}} k_{\mathrm{d}}\right)^{\alpha}(r-1)}{r\left(1+r k_{\mathrm{d}} / k_{\mathrm{u}}\right)} \tilde{\mu}^{-1}\left(U_{\mathrm{sh}, 2} B_{-5} R_{\perp, 2}\right)^{2}$.

Finally, if the shock is supersonic and super-Alfvénic (Eq. (1)) and if $\mathscr{R}>1$ (Eq. (9)), the maximum energy reached by a particle is $E_{\mathrm{max}}=\min \left[E_{\mathrm{loss}}, E_{\mathrm{damp}}, E_{\mathrm{age}}, E_{\mathrm{esc}, \mathrm{u}}, E_{\mathrm{esc}, \mathrm{d}}\right]$.

\section{Potential particle acceleration sites}

In this section, we identify and characterise possible sites of particle acceleration in protostars. In particular, we consider accretion flows in the collapsing envelopes and on protostellar surfaces as well as jets. The required parameters needed to prove the effectiveness of shock acceleration are shown in Table 1.

It is straightforward to verify that Eq. (9) is not fulfilled in envelopes $(\mathscr{R} \ll 1)$. The ionisation fraction and the shock velocity are too small, quenching the particle acceleration. The magnetic field strength is also large enough to produce a subAlfvénic shock. This rules out envelopes as a possible shock acceleration sites. From now on we focus on shocks in jets and on protostellar surfaces.

\subsection{Jets}

Jets are observed at all stages during the evolution of a protostar (e.g. McCaughrean et al. 2002; Reipurth et al. 1997; Watson \& Stapelfeldt 2004). Jet speeds, $v_{\mathrm{fl}}$, are similar for different classes $\left(60-300 \mathrm{~km} \mathrm{~s}^{-1}\right)$ with shock velocities, $v_{\mathrm{sh}}$, of the order of 20-140 $\mathrm{km} \mathrm{s}^{-1}$ (Raga et al. 2002, 2011; Hartigan \& Morse 2007; Agra-Amboage et al. 2011), then $U_{\mathrm{sh}}=40-160 \mathrm{~km} \mathrm{~s}^{-1}$. The neutral density is between $10^{4}$ and $10^{7} \mathrm{~cm}^{-3}$ (Lefloch et al. 2012; Gómez-Ruiz et al. 2012) with temperatures of the order of $10^{4} \mathrm{~K}$ up to about $10^{6} \mathrm{~K}$ (Frank et al. 2014). There is only one theoretical estimate for the magnetic field strength $(B \sim 300-500 \mu \mathrm{G})$ for Class II sources (Teşileanu et al. 2009, 2012). The transverse radius of a jet is about $5 \mathrm{AU}, 10 \mathrm{AU}$, and $50 \mathrm{AU}$ at $15 \mathrm{AU}$, $100 \mathrm{AU}$, and $1000 \mathrm{AU}$ from the source, respectively (Cabrit et al. 2007; Hartigan et al. 2004). The ionisation fraction in Class I

\footnotetext{
2 The factor 4 in the denominator comes from the fact that the diffusion process in the perpendicular direction is in two dimensions.
}

and II are similar, $x \sim 0.05-0.9$ (Nisini et al. 2005; Maurri et al. 2014), while Class 0 jets are mainly molecular (Dionatos et al. 2010).

\subsection{Accretion flows on protostellar surfaces}

We use the computational results of the protostellar collapse of an initially homogeneous cloud core described in Masunaga \& Inutsuka (2000). Their simulation describes the phase of main accretion, when the protostar mass grows because of the steady accretion from the infalling envelope. They give the temporal evolution of temperature, density, and flow velocity which, assuming a stationary shock, is equal to the shock velocity. The radius of the protostar is set to $2 \times 10^{-2} \mathrm{AU}$ and we find that only the last time step of the simulation, corresponding to the end of the main accretion phase, leads to a strong proton acceleration. Parameters are listed in the third row of Table 1.

\section{Maximum energy of the accelerated particles}

For jet shocks we perform a parametric study using the values in the second row of Table 1, assuming a parallel shock, $\eta=10^{-5}$, and $T=10^{4} \mathrm{~K}$. We also consider $\kappa_{\mathrm{u}}=\kappa_{\mathrm{B}}$, which is the most favourable circumstance for accelerating particles (Drury et al. 1983) and we compute $E_{\max }$ for $R_{\mathrm{sh}}=100 \mathrm{AU}$ and $R_{\perp}=10 \mathrm{AU}$. The upper panel of Fig. 1 shows the maximum energy that a shock-accelerated proton can reach. By increasing both $U_{\text {sh }}$ and $B, E_{\max }$ attains higher values up to about $13 \mathrm{GeV}$ for protons. Once the combination of parameters satisfies the condition $\mathscr{R}>1, E_{\max }$ rapidly reaches a constant value, encompassed by the cyan contours in each subplot. In fact, the maximum energy is controlled by $E_{\mathrm{esc}, \mathrm{d}}$ that is independent of both $n$ and $x$. Supposing the magnetic field to have a strong toroidal component, we repeat the calculation for the case of a perpendicular shock finding that $E_{\max }$ decreases by a factor of about 1.3 , since $E_{\mathrm{esc}, \mathrm{d}}^{\perp} / E_{\mathrm{esc}, \mathrm{d}}^{\|} \propto(r+1) /(2 r)^{3}$.

For shocks on protostellar surfaces, we use values in the third row of Table 1 , varying $x$ and $B$. Assuming $\eta=10^{-5}, k_{\mathrm{u}}=1$, and a parallel shock, we find values of $E_{\max }$ for protons up to about $26 \mathrm{GeV}$ for $B \sim 3-10 \mathrm{G}$ (see lower panel of Fig. 1), which are comparable with magnetic field intensities computed by e.g. Garcia et al. (2001). As a result of high temperatures, Coulomb losses are dominant and $E_{\max }$ is constrained by $E_{\text {loss }}$. Thus, for a perpendicular shock, $E_{\max }$ is a factor of about 1.3 larger, since $E_{\text {loss }}^{\perp} / E_{\text {loss }}^{\|} \propto(r+1) / r$. Hatched areas in both panels of Fig. 1 show regions where acceleration is not possible because of strong wave damping.

Electrons can be accelerated as well, but generally $E_{\max }$ for electrons is much smaller than $E_{\max }$ for protons because of wave damping and stronger energy losses. For instance, for $U_{\mathrm{sh}}=160 \mathrm{~km} \mathrm{~s}^{-1}$ and $B=1 \mathrm{mG}, E_{\max } \sim 300 \mathrm{MeV}$ for a narrow range of density and ionisation fraction $\left(n \gtrsim 3 \times 10^{6} \mathrm{~cm}^{-3}\right.$, $x \gtrsim 0.6$ ). For lower values of $B$ and $U_{\text {sh }}, E_{\text {max }} \lesssim 50 \mathrm{MeV}$. We also find that electron acceleration is not triggered by protostellar surface shocks.

\section{Discussion and conclusions}

We investigated the possibility of accelerating particles within a protostellar source by means of shock processes through the

\footnotetext{
3 Superscripts $\perp$ and $\|$ refer to perpendicular and parallel shocks, respectively.
} 

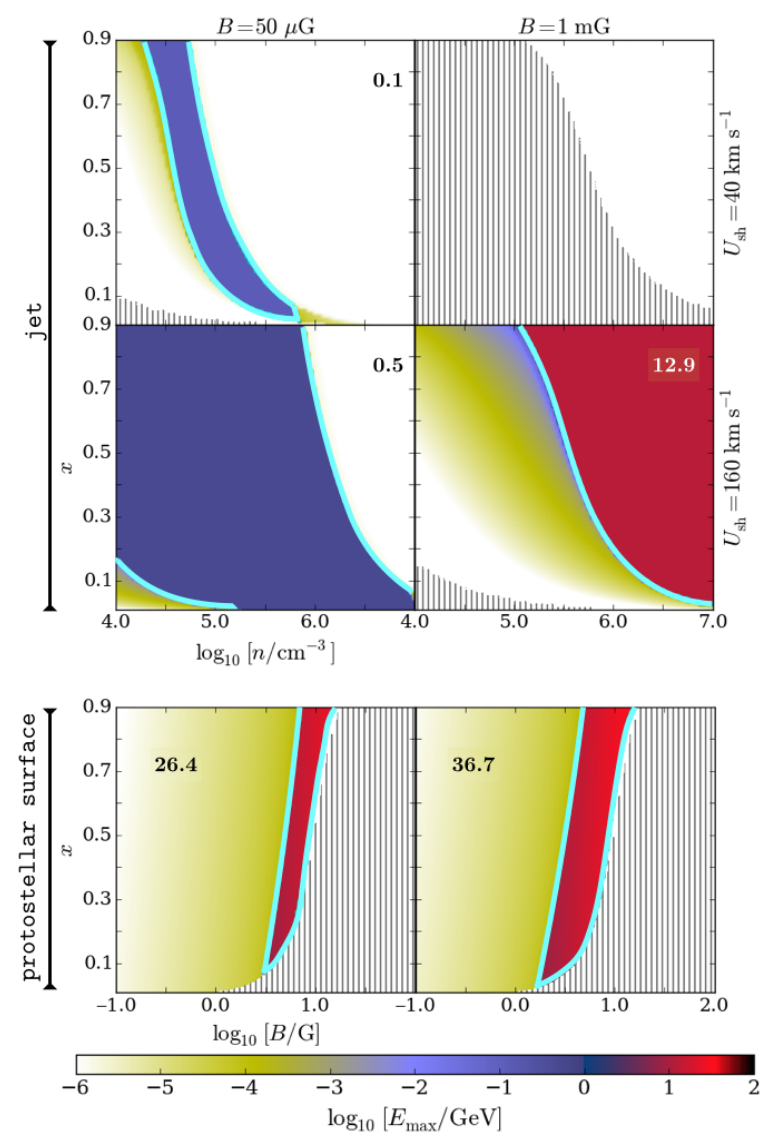

Fig. 1. Upper panel: case of a parallel shock in jets: ionisation fraction, $x$, versus density of neutrals, $n$, for $U_{\mathrm{sh}}=40$ and $160 \mathrm{~km} \mathrm{~s}^{-1}$, $B=50 \mu \mathrm{G}$ and $1 \mathrm{mG}$, and $T=10^{4} \mathrm{~K}$. Lower panel: case of parallel (left) and perpendicular (right) shocks on protostellar surfaces: ionisation fraction, $x$, versus magnetic field strength, $B$, for $U_{\mathrm{sh}}=260 \mathrm{~km} \mathrm{~s}^{-1}$, $T=9.4 \times 10^{5} \mathrm{~K}$, and $n=1.9 \times 10^{12} \mathrm{~cm}^{-3}$. Colour maps show values of $E_{\max }$ for protons and cyan contours delimit the regions where $E_{\max }$ reaches its maximum constant value shown in $\mathrm{GeV}$ in each subplot. Vertically hatched regions refer to combinations of parameters corresponding to strong wave damping $(\mathscr{R}<1)$.

diffusive shock acceleration mechanism. We focused our attention on the effectiveness of shocks in envelopes, on protostellar surfaces, and in jets. We concluded that:

(i) In envelopes, $x$ and $U_{\text {sh }}$ are too small, preventing any particle acceleration. Besides, $B$ is as large as to yield sub-Alfvénic shocks.

(ii) Jet shocks are possible accelerators of particles that can be easily boosted up to relativistic energies. The acceleration is more efficient for protons which can reach up to about 13 or $10 \mathrm{GeV}$ (for parallel or perpendicular shocks, respectively), while electrons attain at most about $300 \mathrm{MeV}$ because of wave damping and energy losses.

(iii) Protostellar surface shocks can accelerate protons up to about 26 or $37 \mathrm{GeV}$ (for parallel or perpendicular shocks, respectively). Electrons cannot be accelerated mainly because of large magnetic field strengths leading to synchrotron losses.

The set of conditions that has to be fulfilled is highly non-linear: small variations in one or more parameters $\left(B, x, n, T, U_{\mathrm{sh}}, \eta\right.$, $k_{\mathrm{u}}, k_{\mathrm{d}}$ ) can make the acceleration process inefficient. As a consequence, since young protostars are highly dynamic systems, particle acceleration can be a very intermittent process. In a subsequent paper, we will discuss in detail other possible acceleration mechanisms as well as the effect of variations in the parameter set and the distance of the jet shocks from the protostar, including departures from Bohm-type diffusion with the aim of studying the propagation of high-energy particles in the protostellar environment to explain the available observations.

Acknowledgements. We thank Elena Amato, Francesca Bacciotti, Sylvie Cabrit, Claudio Codella, Daniele Galli, and Linda Podio for valuable discussions about jets in protostars and shock properties. We also thank the anonymous referee for a careful reading and thoughtful comments. We acknowledge the financial support of the Agence National pour la Recherche (ANR) through the COSMIS project. This work has been carried out thanks to the support of the OCEVU Labex (ANR-11-LABX-0060) and the A*MIDEX project (ANR-11-IDEX-0001-02) funded by the "Investissements d'Avenir" French government programme managed by the ANR. M.P. and A.M. also acknowledge the support of the CNRSINAF PICS project "Pulsar wind nebulae, supernova remnants and the origin of cosmic rays".

\section{References}

Agra-Amboage, V., Dougados, C., Cabrit, S., \& Reunanen, J. 2011, A\&A, 532, A59

Ainsworth, R. E., Scaife, A. M. M., Ray, T. P., et al. 2014, ApJ, 792, 18

Balbus, S. A., \& Hawley, J. F. 1991, ApJ, 376, 214

Bell, A. R. 1978, MNRAS, 182, 147

Berezhko, E. G. 1996, Astropart. Phys., 5, 367

Bykov, A. M. 2004, Adv. Space Rev., 33, 366

Cabrit, S., Codella, C., Gueth, F., et al. 2007, A\&A, 468, L29

Ceccarelli, C., Dominik, C., López-Sepulcre, A., et al. 2014, ApJ, 790, L1

Cleeves, L. I., Adams, F. C., \& Bergin, E. A. 2013, ApJ, 772, 5

de Gouveia Dal Pino, E. M. 1995, AIP Conf. Ser., 345, 427

Dionatos, O., Nisini, B., Cabrit, S., Kristensen, L., \& Pineau Des Forêts, G. 2010, A\&A, 521, A7

Drury, L. O’C. 1983, Rep. Prog. Phys., 46, 973

Drury, L. O'C., Duffy, P., \& Kirk, J. G. 1996, A\&A, 309, 1002

Duley, W. W., \& Williams, D. A. 1984, Interstellar Chemistry (Academic Press) Frank, A., Ray, T. P., Cabrit, S., et al. 2014, Protostars and Planets VI, eds. H. Beuther, R. S. Klessen, C. P. Dullemond, \& Th. Henning (Tucson: University of Arizona Press), 451

Garcia, P. J. V., Ferreira, J., Cabrit, S., \& Binette, L. 2001, A\&A, 377, 589

Gómez-Ruiz, A. I., Gusdorf, A., Leurini, S., et al. 2012, A\&A, 542, L9

Hartigan, P., \& Morse, J. 2007, ApJ, 660, 426

Hartigan, P., Edwards, S., \& Pierson, R. 2004, ApJ, 609, 261

Kirk, J. G. 1994, in Plasma Astrophysics, eds. A. O. Benz, \& T., J.-L. Courvoisier (Berlin: Springer Verlag), 225

Lefloch, B., Cabrit, S., Busquet, G., et al. 2012, ApJ, 757, 25

Mannheim, K., \& Schlickeiser, R. 1994, A\&A, 286, 983

Masunaga, H., \& Inutsuka, S. 2000, ApJ, 531, 350

Maurri, L., Bacciotti, F., Podio, L., et al. 2014, A\&A, 565, A110

McCaughrean, M., Zinnecker, H., Andersen, M., Meeus, G., \& Lodieu, N. 2002, The Messenger, 109, 28

Nisini, B., Bacciotti, F., Giannini, T., et al. 2005, A\&A, 441, 159

Padovani, M., \& Galli, D. 2011, A\&A, 530, A109

Padovani, M., \& Galli, D. 2013, in Cosmic Rays in Star-Forming Environments, eds. D. F. Torres, \& O. Reimer, ASSP, 34, 61

Padovani, M., Galli, D., \& Glassgold, A. E. 2009, A\&A, 501, 619

Padovani, M., Hennebelle, P., \& Galli, D. 2013, A\&A, 560, A114

Padovani, M., Galli, D., Hennebelle, P., Commerçon, B., \& Joos, M. 2014, A\&A, 571, A33

Podio, L., Lefloch, B., Ceccarelli, C., Codella, C., \& Bachiller, R. 2014, A\&A, 565, A64

Raga, A. C., Velázquez, P. F., Cantó, J., \& Masciadri, E. 2002, A\&A, 395, 647

Raga, A. C., Noriega-Crespo, A., Lora, V., Stapelfeldt, K. R., \& Carey, S. J. 2011, ApJ, 730, 17

Reipurth, B., Hartigan, P., Heathcote, S., Morse, J. A., \& Bally, J. 1997, AJ, 114, 757

Schlickeiser, R. 2002, Cosmic Ray Astrophysics (Berlin, Heidelberg: SpringerVerlag)

Swartz, W. E., Nisbet, J. S., \& Green, A. E. S. 1971, J. Geophys. Res., 76, 8425

Teşileanu, O., Massaglia, S., Mignone, A., Bodo, G., \& Bacciotti, F. 2009, A\&A, 507, 581

Teşileanu, O., Mignone, A., Massaglia, S., \& Bacciotti, F. 2012, ApJ, 746, 96

Watson, A. M., \& Stapelfeldt, K. R. 2004, ApJ, 602, 860 